\title{
Delayed Leukoencephalopathy: Three Case Reports and a Literature Review
}

\author{
Ángel Torralba-Morón ${ }^{1}$, Juan Ortiz-Imedio ${ }^{1}$, Montserrat Morales-Conejo ${ }^{1}$, Juan Ruiz-Morales², Juan-Manuel Guerra-Vales ${ }^{1}$ \\ ${ }^{1}$ Internal Medicine Department, University Hospital 12 de Octubre, Madrid, Spain \\ ${ }^{2}$ Neurology Department, University Hospital 12 de Octubre, Madrid, Spain
}

Received: 03/10/2016

Accepted: 08/11/2016

Published: $30 / 11 / 2016$

How to cite this article: Torralba-Moron A, Ortiz-Imedio J, Morales-Conejo M, Ruiz-Morales J, Guerra-Vales JM. Delayed leukoencephalopathy: three case reports and a literature review. EJCRIM 2016;3: doi:10.12890/2016_000511.

Conflicts of Interests: The Authors declare that there are no competing interests.

This article is licensed under a Commons Attribution Non-Commercial 4.0 License

\section{ABSTRACT}

Background: Delayed leukoencephalopathy $(\mathrm{DL})$ is a rare entity associated with cerebral hypoxia and heroin consumption. We describe the clinical course of three cases of DL due to non-heroin drug use.

Material and methods: We describe the cases of three DL patients admitted to our hospital in 2012.

Discussion: These cases contribute to the aetiological spectrum of DL since multifactorial causes could account for the clinical symptoms.

\section{LEARNING POINTS}

- Substances toxic to the CNS can damage the CNS directly (direct toxicity) or by depressing the respiratory centre (cerebral hypoxia).

- As clinical manifestations can appear after a time lag, we recommend a period of initial monitoring.

- Histological and radiological findings can contribute to better understanding of the pathophysiological mechanisms and causes involved.

\section{KEYWORDS}

delayed leukoencephalopathy, globus pallidus, methadone

\section{BACKGROUND}

Delayed leukoencephalopathy (DL) is caused by structural injury to the white matter of the central nervous system (CNS), with a characteristic two-phase clinical course: recovery from an episode of low level of awareness, caused by acute or chronic neurological damage, is followed by neuropsychiatric symptoms which include cognitive and level of consciousness disorders, behavioural disorders and dysautonomy. The disorder is associated with parkinsonism when grey matter basal nuclei are involved ${ }^{[1]}$.

The condition was initially called delayed post-hypoxic leukoencephalopathy (DPHL) and correlated with causes of cerebral hypoxia, such as CO poisoning or respiratory depression due to asphyxia, constriction, drugs, etc. ${ }^{[2,3]}$. In DL, the grey matter basal nuclei are damaged by hypoxia ${ }^{[1]}$, the posterior fossa structures are not affected, and there is increased permeability of the blood-brain barrier ${ }^{[4]}$. There is severe diffuse demyelination, but the subcortical U-fibres are not damaged, and neither spongiosis nor vacuolation occur ${ }^{[3]}$. The disease usually has a good prognosis.

Leukoencephalopathy following heroin pyrolysate inhalation predominantly affects the cerebellum and brainstem. The condition presents with two gradual clinical phases, without an intervening recovery period. During the initial stage the disease causes cerebellar ataxia, hyperactive deep tendon reflexes, hypertonic hemiplegia or tetraplegia, choreoathetoid movements and pseudobulbar reflexes. In the 
terminal stage, which does not always develop, it causes central pyrexia, spasms, hypotonic paresis, areflexia and akinetic mutism. Specific histological findings include spongiform degeneration of the CNS white matter with vacuoles and cavities, foci of necrosis in the spongiform white matter and multivacuolar degeneration of the oligodendroglia and axons, all of which affect the hemispheres, cerebellum, brainstem and spinal cord ${ }^{[5]}$.

Isolated cases with a similar neurological picture, attributable to consumption of methadone and other toxic substances (benzodiazepines, neuroleptics, barbiturates, etc.), demonstrating a low level of consciousness and possible associated hypoxia, have been reported ${ }^{[2]}$.

We present the case reports below to increase knowledge of the aetiological spectrum of DL syndromes. A review of the literature on the subject is also provided.

\section{CASE REPORT}

Case 1 was a 42-year-old male polydrug user (but no heroin use for over 5 years). After a methadone, alprazolam and alcohol overdose, he experienced an episode of low level of consciousness, respiratory depression and fever without clinical focus. Initial neuroimaging revealed non-specific findings.

The patient's consciousness level initially recovered but suddenly worsened 13 days later. During the following weeks, he remained febrile, with spontaneous resolution, and with fluctuating consciousness, which gradually improved. He had widespread myoclonus. Cranial MRI (Fig. 1) revealed bilateral, symmetrical and diffuse involvement of the cerebral white matter, as well as bilateral globus pallidus injury. EEG revealed diffuse slowing of background neuronal activity. The patient remained stable. Lack of attention and dysexecutive and amnestic abnormalities persisted at discharge.

Case 2 was a 43-year-old man living with HIV treated with emtricitabine/tenofovir and lopinavir/ritonavir, HCV genotype 3 chronic liver disease and drug abuse (no heroin use for over 5 years). He was admitted after a methadone overdose with a low level of consciousness and a bradypnoea episode. This was associated with tachycardia and sweating, as well as fever without clinical focus, which resolved spontaneously. An initial cranial CT scan revealed decreased image density in both globus pallidi.

During the first few days, the patient recovered awareness and made spontaneous voluntary movements. He reacted by opening his eyes when spoken to and answered with basic words. However, drowsiness, slow thinking and occasional agitation remained.

During the following days, the low level of awareness, sweating, tachypnoea and fever reappeared. Associated infection was not observed. A chest angio-CT ruled out pulmonary embolism. The patient did not speak, and presented spastic rigidity and an extensor motor response to pain. Neuroimaging revealed bilateral, symmetrical and diffuse involvement of the cerebral white matter, with clinical progression of the lesions in both globus pallidi (Fig. 2). EEG revealed diffuse slowing of background neuronal activity. A cerebrospinal fluid study was normal. The patient gradually deteriorated without recovering consciousness. Tachypnoea, fever and sweating remained. He died 40 days after the onset of symptoms.

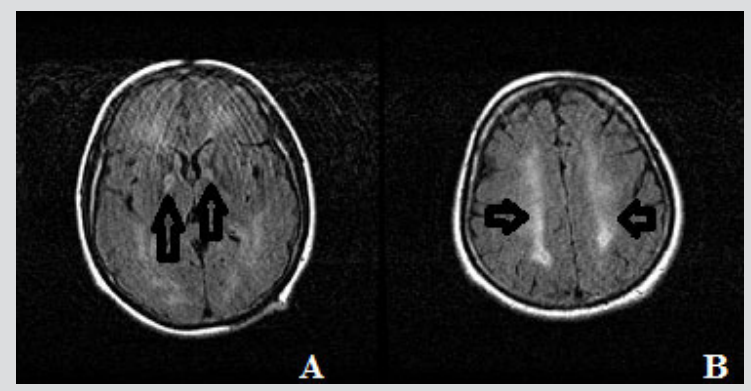

Figure 1. Cranial MRI. (A) Hypointense lesions in both globus pallidi (arrows). (B) Bilateral symmetrical hyperintense white matter lesions (arrows)

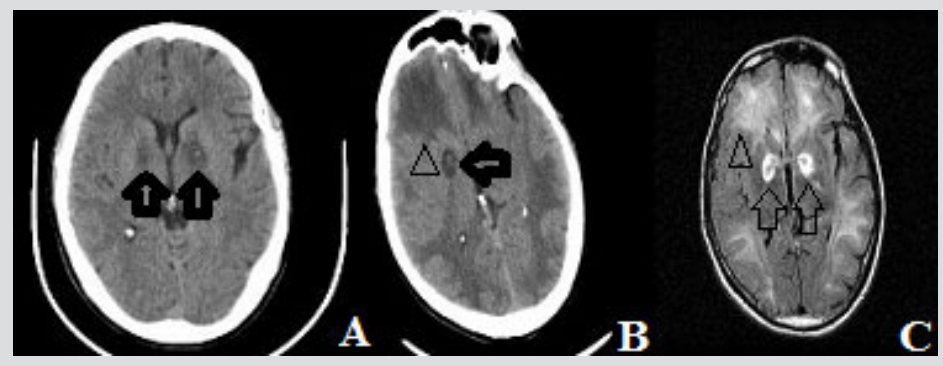

Figure 2. (A, B) Cranial CT showing hyperintense lesions in globus pallidi (arrows) and a hypointense white matter lesion (arrowhead). (C) Cranial MRI showing hypointense lesions in globus pallidi, with peripheral hyperintense (arrow) and diffuse, bilateral and symmetrical hyperintense white matter lesions (arrowhead) 


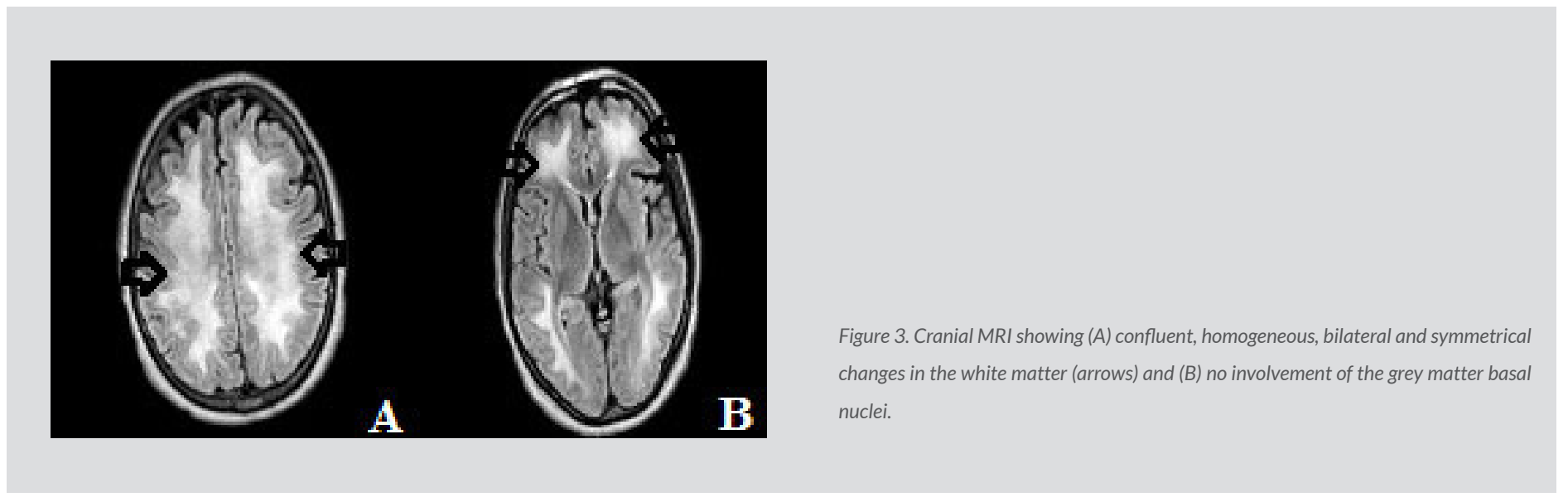

Case 3 was a 52-year-old male polydrug user (no heroin use for over 5 years), living with HIV treated with emtricitabine/tenofovir and lopinavir/ritonavir and chronic liver disease secondary to HBV/HDV coinfection. He was admitted for abnormal behaviour, disorientation, inattention and low level of consciousness after intoxication with venlafaxine and alprazolam. There were no findings on initial cranial CT. Over the following days, the patient regained consciousness and stayed alert, inattentive and with hyperreflexia. Brain MRI revealed homogeneous, bilateral and symmetrical changesin the intensity of the cerebral white matter (Fig. 3). EEG showed slow background baseline activity. There were no abnormalities in the cerebrospinal fluid study.

The patient gradually showed a better level of consciousness, maintained a coherent conversation and obeyed simple commands. He achieved independent gait. At discharge, he remained disoriented, with disturbed immediate memory and motor apraxia.

\section{DISCUSSION}

In all three cases reported, the patient underwent an episode of low level of consciousness, followed by initial improvement and then further development of leukoencephalopathy symptoms. This biphasic clinical course indicates all three patients had DL. Motor symptoms may follow globus pallidus injury, but were not present in case 3. Damage to the cerebral grey matter suggests cerebral hypoxia, which is a rare finding in the pure toxic leukoencephalopathies ${ }^{[4]}$.

These patients had not used heroin for at least 5 years and no cerebellar involvement was shown by the neuroimaging tests. Two of the three patients also experienced a recovery period between the two clinical phases of the disease, but this temporary recovery was not seen in the leukoencephalopathy caused by heroin inhalation ${ }^{[5]}$.

The time lag between the two clinical phases of the disease could be due to oligodendrocyte injury, which prevents replacement of myelin in the CNS and causes late onset demyelination ${ }^{[3]}$.

These case reports contribute to the possible aetiology of this rare disease, since the drugs involved can cause both direct brain toxicity and cerebral hypoxia through depression of the respiratory centre. The mentioned drugs should be included in the differential diagnosis of DL. Their mechanisms of action can cause both DPHL and delayed toxic leukoencephalopathy. It is also thought that both conditions can sometimes co-exist.

\section{REFERENCES}

1. Filley C, Kleinschmidt-DeMasters BK. Toxic leukoencephalopathy. N Engl J Med 2001;345:425-432.

2. Carroll I, Heritier AC, Dirren E, Burkhard PR, Horvath J. Delayed leukoencephalopathy after alprazolam and methadone overdose: a case report and review of the literature. Clin Neurol Neurosurg 2012;114:816-819.

3. Shprecher D, Mehta L. The syndrome of delayed post-hypoxic leukoencephalopathy. NeuroRehabilitation 2010;26:65-72.

4. Cerase A, Leonini S, Bellini M, Chianese G, Venturi C. Methadone-induced toxic leukoencephalopathy: diagnosis and follow-up by magnetic resonance imaging including diffusion-weighted imaging and apparent diffusion coefficient maps. J Neuroimaging 2011;21:283-286.

5. Wolters EC, van Wijngaarden GK, Stam FC, Rengelink H, Lousberg RJ, Schipper ME et al. Leucoencephalopathy after inhaling heroin pyrolysate. Lancet 1982;2:1233-1237. 